\title{
Compact and Energy Efficient Neuron With Tunable Spiking Frequency in 22-nm FDSOI
}

\author{
Hossein Eslahi, Graduate Student Member, IEEE, Tara J. Hamilton, Member IEEE, and Sourabh Khandelwal, \\ Senior Member IEEE
}

\begin{abstract}
In this paper, we present a mixed-signal integrate and fire neuron designed in a 22-nm FDSOI technology. In this novel design, we deploy the back-gate terminal of FDSOI technology for a tunable design. For the first time, we show analytically and with pre- and post-layout simulations a neuron with tunable spiking frequency using the back-gate voltage of FDSOI technology. The neuron circuit is designed in the subthreshold region and dissipates an ultra-low energy per spike of the order of Femto Joules per spike. With the layout area of only $30 \mu \mathrm{m}^{2}$, this is the smallest neuron circuit reported to date.
\end{abstract}

Index Terms-Spiking Neural Networks, Integrated Circuits, Neuromorphic Computing, Neuron and Synapse, FDSOI, Frequency Modulation.

\section{INTRODUCTION}

Artificial neural networks (ANN) are a promising solution to address the bottlenecks of the Von Neumann paradigms [1]-[18]. Brain-inspired spiking neural networks (SNN) have been introduced as the third generation of ANN emulating biological brain functions in hardware. The basic computing unit in the spiking neural networks is neurons communicating with each other through synapses via the electrical pulses called spikes [2]-[6], [9]-[16], [19]-[24].

To mimic brain-like functionality a very large number of neurons are required in SNNs. So, the size of the neuronal hardware is also very important. This underlines the need for simple neuronal circuits with minimum number of transistors. The Axon-Hillock [25], Hodgkin-Huxley [26], Morris-Lecar [27], Fitz-Nagumo [28], Resonate and Fire [29], [30], Hindmarsh-Rose [31], Mihalas-Niebur and Izhikevich model [32][36] were the leading models inspiring the next generations of the neuromorphic circuit over the last decades [1], [2], [4], [7], [15], [18], [22], [24]. Among these, the Leaky Integrate-and-Fire $(\mathrm{I} \& \mathrm{~F})$ neurons are claimed to be compact, and computationally more efficient. The I\&F neurons integrate the pre-synaptic signal through a membrane capacitor and generate spikes once the integrated signal exceeds the spiking threshold [3], [5], [9], [12]-[17], [19]-[21], [23], [32], [37][40].

To address the energy issue, the transistors are preferably biased in the sub-threshold region. However, nano-scale CMOS devices suffer from significant leakage currents in sub-threshold. Therefore, novel technologies like the Fully Depleted Silicon on Insulator (FDSOI) have been emerged showing superior performance in terms of leakage and power consumption [1], [16], [18], [41], [42]. The use of back-gate bias is a new possibility in the FDSOI technology to tune the circuit performance. In this paper, for the first time, we propose a neuron design which can use back-gate voltage to modulate the spiking frequency of a compact I\&F neuron without affecting the leakage. This tunability via back-gate control is compatible with the mixed signal approach where the back-gate voltage can be generated with on-chip DACs. The neuron operates in the sub-threshold region and consumes an ultra-low energy per spike of the order of few Femto Joules. The final layout, including the core neuron, six output buffers for measurement purposes, one level-shifter (required for the following I/O block), and all capacitors, is only $30 \mu^{2}$ in size.

The paper is organized as follows. Neuron circuit topology and its functionality are presented in the next section. An analytical model of the frequency modulation as well as the temperature compensation based on the back-gate biasing are also presented in section II. In Section III, the postlayout analysis and the Monte-Carlo simulation results are presented, validating the neuron performance against the process variations, mismatch, leakage, and parasitic components. Conclusions are drawn in the Section IV.

\section{FDSOI-BASED I\&F NEURON AND FREQUENCY MODUlation}

\section{A. Neuron Operation}

The proposed Integrate-and-Fire neuron is designed in 22nm FDSOI technology. The topology is shown in Fig. 1. The supply voltage $V_{d d}$ is set to $0.3 \mathrm{~V}$ for the sub-threshold operation. The input synaptic current $I_{\text {syn }}$ is a fixed bias current. It is set it to $10 n A$. Each spike consists of three main transient responses: charging, firing, and resetting. The proposed neuron performs these steps through different parts highlighted in black, green, and red in Figs. 1(a)-(b), respectively. Figure 2 correspondingly illustrate the contribution of each step in the simplified characteristic of the spikes with the same colour.

The charging time depends on the membrane capacitance $C_{m e m}$ and the difference between the synaptic current $I_{s y n}$ and the current provided by the After Hyper-Polarization (AHP) mechanism which composes the transistors $M_{4-6}$ and capacitor $C_{a h p}$, shown in black color in Fig. 1(a). The AHP mechanism is also used to modulate the spiking-frequency. $C_{m e m}$ is responsible for integrating the difference of the synaptic current $I_{s y n}$ and the AHP current $I_{a h p}$ and, then, creating the membrane voltage $V_{\text {mem }}$. The charging starts when $I_{\text {syn }}>I_{a h p}$. The membrane voltage increases from zero at point $\mathrm{A}$ in Fig.2 and the charging continues until $V_{m e m}$ reaches the spiking threshold $V_{t h-s}$ at point B. This step, therefore, takes a charging time $t_{A B}$ that is a function of $C_{m e m}, I_{s y n}$, and $I_{a h p} . t_{A B}$ can be calculated by integrating the membrane voltage from zero to the spiking threshold as, 

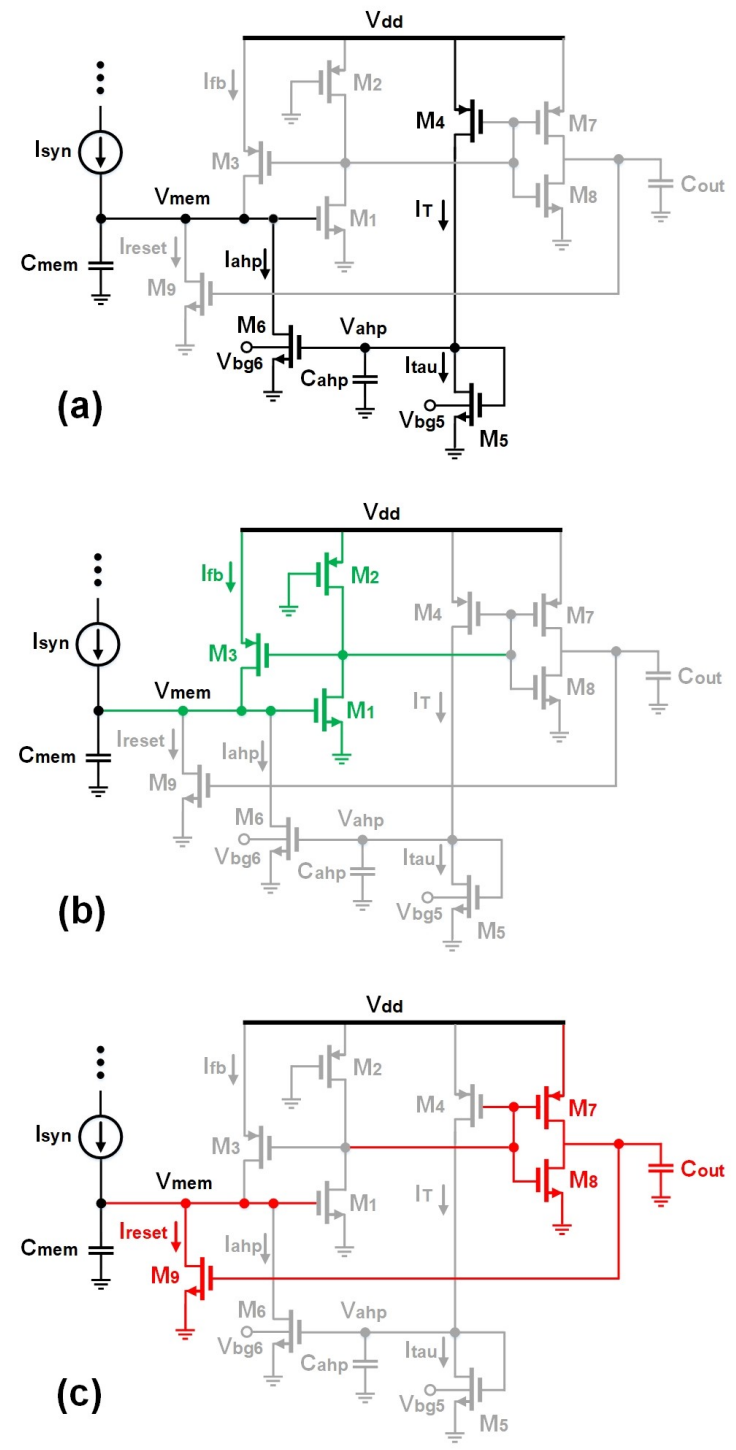

Fig. 1. The proposed I\&F neuron with frequency adaptation mechanism. The black (a), green (b), and red (c) parts are respectively responsible for the charging, firing, and resetting steps.

$$
\int_{0}^{V_{t h-s}} C_{m e m} d V_{m e m}=\int_{0}^{t_{A B}}\left(I_{s y n}-I_{a h p}\right) d t
$$

Assuming a fixed pre-synaptic current $I_{s y n}$, the charging time $t_{A B}$ is obtained to be,

$$
t_{A B}=\frac{C_{m e m} \cdot V_{t h-s}}{I_{s y n}-I_{a h p}}
$$

Once $V_{\text {mem }}$ exceeds the spiking threshold, transistors $M_{1}$ turns on. This then turns on $M_{3}$. Then, a firing current $I_{f b}$ flows through $M_{3}$ and adds extra charges to $C_{m e m}$, pulling up the membrane voltage $V_{m e m}$ to the supply voltage $V_{d d}$ (See point $\mathrm{C}$ in Fig. 2). This current is, therefore, responsible for generating the spikes. The green part in Fig. 1(b) shows the active part of the neuron in the firing time. The membrane voltage changes from $V_{t h-s}$ to $V_{d d}$ in the firing step. The firing time $t_{B C}$ is calculated as,

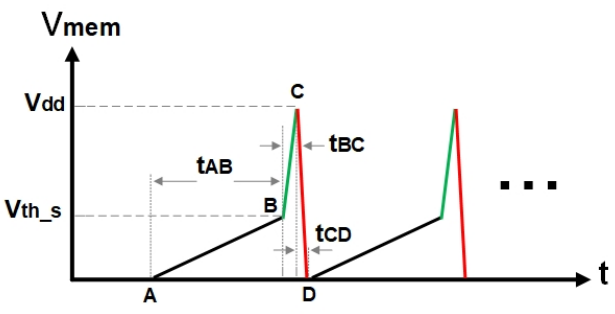

Fig. 2. Simplified spiking characteristic of the neuron in Fig. 1. Each spike composes three steps named the charging (from A to B), firing (between points $\mathrm{B}$ and $\mathrm{C}$ ), and resetting (from $\mathrm{C}$ to $\mathrm{D}$ ).

$$
t_{B C}=\frac{C_{m e m} \cdot\left(V_{d d}-V_{t h-s}\right)}{I_{s y n}-I_{a h p}+I_{f b}} \approx \frac{C_{m e m} \cdot\left(V_{d d}-V_{t h-s}\right)}{I_{f b}}
$$

As soon as $M_{1}$ switches on, the inverter $\left(M_{7}\right.$ and $\left.M_{8}\right)$ sees a zero bias as its input. As a result the output capacitor $C_{\text {out }}$ starts to charge. The output capacitance should be greater than the membrane capacitance to guarantee the sequence of firing and resetting steps. Once $C_{\text {out }}$ charges to voltage which can turn $M_{9}$ on, the reset current $I_{\text {reset }}$ starts to flow (Fig.1(c)). $I_{\text {reset }}$ discharges the membrane capacitor to point D in Fig.2 (zero volt in our design). The $\Delta V_{m e m}$ in this step is $V_{d d}$ and the resetting time $t_{C D}$ is given by,

$$
t_{C D}=\frac{C_{\text {out }} \cdot V_{d d}}{I_{\text {syn }}-I_{a h p}-I_{\text {reset }}} \approx \frac{C_{\text {out }} \cdot V_{d d}}{I_{\text {reset }}}
$$

The neuron remains at this point as long as $I_{\text {reset }}>$ $I_{\text {syn }}-I_{a h p}$. Subsequently $M_{1}$ switches off and the inverter is connected to $V_{d d}$ through $M_{2}$. Then the next charging starts. $t_{B C}$ and $t_{C D}$ are very small due to the relatively high charging currents $I_{f b}$ and $I_{\text {reset }}$.

\section{B. Frequency Modulation}

The total period of each spike is the summation of $t_{A B}$, $t_{B C}$, and $t_{C D}$. The spiking frequency $f_{\text {spike }}$ is, then, defined as

$$
f_{\text {spike }}=\frac{1}{t_{A B}+t_{B C}+t_{C D}}
$$

Note that the firing and resetting currents are constant, so, $t_{B C}$ and $t_{C D}$ do not change by back-gate biases $V_{b g 5}$ and $V_{b g 6}$. Also, these two time steps are shorter than $t_{A B}$. To modulate the frequency, we modulate $t_{A B}$ through the AHP current $I_{a h p}$. FDSOI devices offer unique way to modulate the device current through the back-gate. Figure 3 plots the drain current versus gate voltage $\left(I_{d}-V_{g}\right)$ characteristics of n- and p-types FDSOI devices with the channel width and length of $W=1.28 \mu \mathrm{m}$ and $L=20 \mathrm{~nm}$, respectively, and the drain voltage $V_{d}=1.5 \mathrm{~V}$. The back-gate voltage $V_{b g}$ is set to $-2.5 \mathrm{~V}$, $0 \mathrm{~V}$, and $2.5 \mathrm{~V}$. As seen, the higher $V_{b g}$, the smaller threshold voltage, and the greater drain current will be. We use the backgate bias voltage over of $M_{6}$ to change $I_{a h p}$ and, subsequently, the charging time $t_{A B}$. The back-gate voltages $V_{b g 5}$ and $V_{b g 6}$ are used to tune the AHP current. Our analytical calculations are validated by comparing with the simulations of $t_{A B}$ with the foundry PDK. 


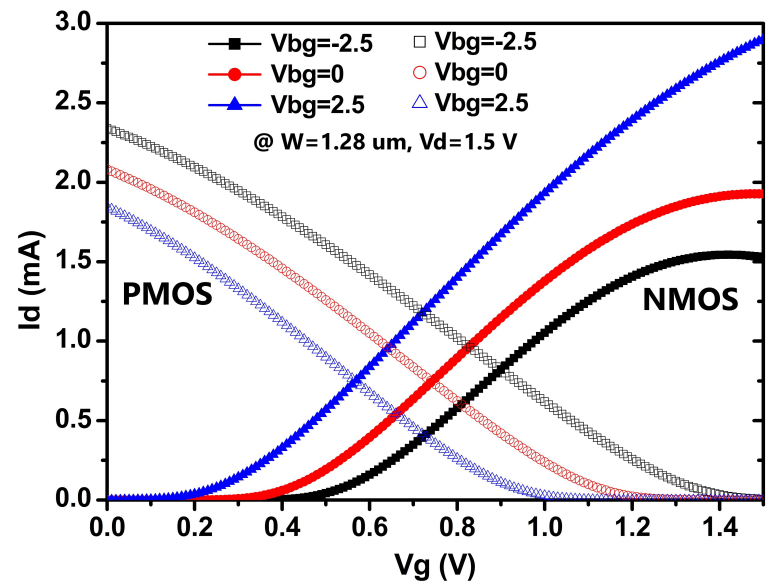

Fig. 3. $I_{d}-V_{g}$ characteristics of two NMOS and PMOS device with the same channel width $W=1.28 \mu \mathrm{m}$ as functions of their back-gate bias.

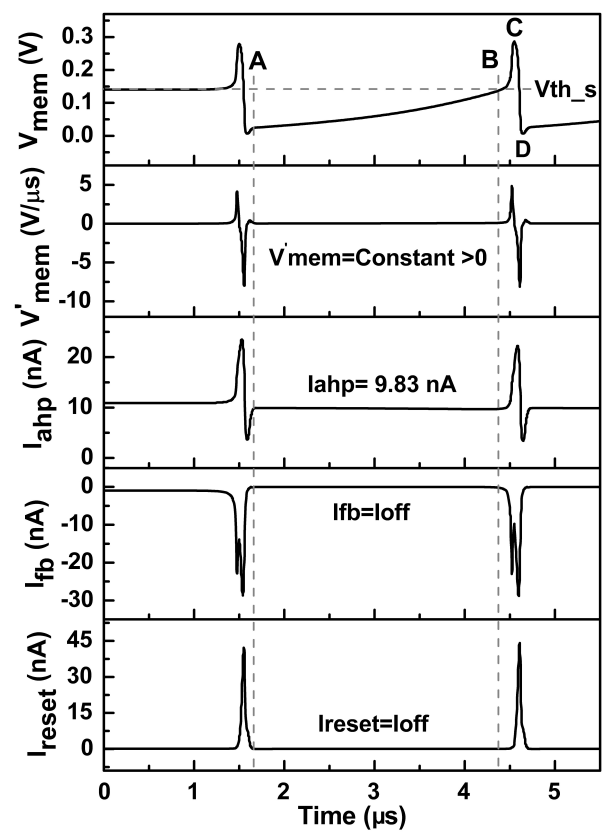

Fig. 4. Calculation of the charging time $t_{A B}$ for the back-gate voltages of $V_{b g 5}=0 \mathrm{~V}$ and $V_{b g 6}=1.2 \mathrm{~V}$. The charging, firing, and resetting steps correspond to the time intervals of $\mathrm{A}$ to $\mathrm{B}, \mathrm{B}$ to $\mathrm{C}$, and $\mathrm{C}$ to $\mathrm{D}$, respectively.

The capacitors are designed by considering the sequence of the charging, firing, and resetting steps. To ensure appropriate sequencing we use $C_{\text {mem }}=3 \mathrm{fF}, C_{\text {out }}=9 \mathrm{fF}$, and $C_{a h p}=40 \mathrm{fF}$. Back-gate voltages are tuned between the allowed range of $0 \mathrm{~V}$ to $1.2 \mathrm{~V}$. Figure 4 sketches the transient characteristics of $V_{m e m}$, derivative of $V_{m e m}\left(V_{m e m}^{\prime}\right), I_{a h p}$, $I_{f b}$, and $I_{\text {reset }}$ for $V_{b g 5}=0 \mathrm{~V}$ and $V_{b g 6}=1.2 \mathrm{~V}$. This is the back-gate voltage condition of the lowest spiking frequency.

As shown in Fig. $4 I_{f b}$ and $I_{\text {reset }}$ are equal to the offcurrent $I_{o f f}$ and $I_{a h p}$ is constant during the charging period. The rate of charging remains roughly constant from point $\mathrm{A}$ to B. However, the firing current $I_{f b}$ starts increasing at point $\mathrm{B}$ and $V_{m e m}^{\prime}$ increases. This indicates that the charging step is terminated and the firing step has started.

The maximum $V_{\text {mem }}$ in the charging step (at point $\mathrm{B}$ ) defines the spiking threshold $V_{t h-s}$. Figure 5 provides easier visualization of the spiking threshold. Fig. 5 shows transient

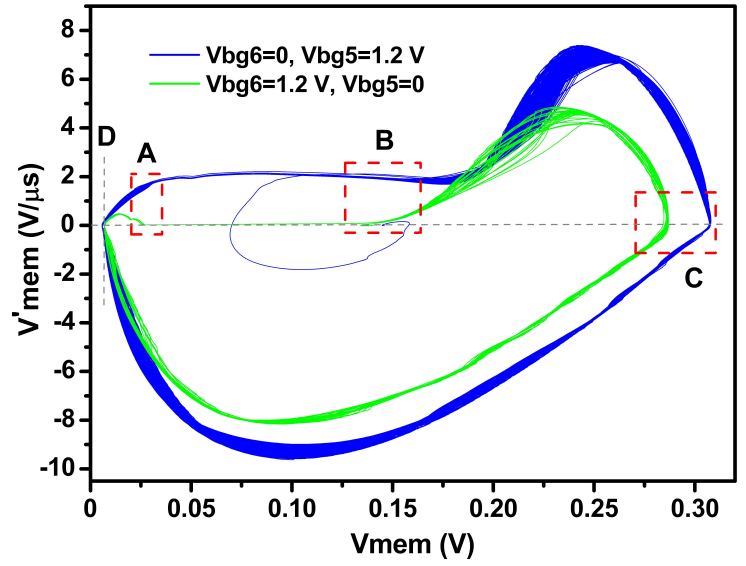

Fig. 5. $V^{\prime}$ mem versus the membrane voltage for two opposite case of the maximum (blue) and minimum (green) spiking frequencies. Three red boxes highlight the corresponding areas of the points $\mathrm{A}, \mathrm{B}$, and $\mathrm{C}$. The part from point $\mathrm{A}$ to point $\mathrm{B}$ indicates a relatively fixed slope.

TABLE I

COMPARISON BETWEEN THE CALCULATED AND SIMULATED SPIKING FREQUENCY FOR DIFFERENT COMBINATIONS OF $V_{b g 5}$ AND $V_{b g 6}$

\begin{tabular}{|c|c|c|c|c|c|}
\hline \multicolumn{2}{|c|}{} & Case 1 & Case 2 & Case 3 & Case 4 \\
\cline { 3 - 6 } \multicolumn{2}{|c|}{} & $V_{b g 5}=1.2 \mathrm{~V}$ & $V_{b g 5}=0 \mathrm{~V}$ & $V_{b g 5}=0 \mathrm{~V}$ & $V_{b g 5}=1.2 \mathrm{~V}$ \\
& $V_{b g 6}=1.2 \mathrm{~V}$ & $V_{b g 6}=0 \mathrm{~V}$ & $V_{b g 6}=1.2 \mathrm{~V}$ & $V_{b g 6}=0 \mathrm{~V}$ \\
\hline \multirow{2}{*}{$f_{\text {spike }}$} & Calculation & $2.5 \mathrm{MHz}$ & $3.2 \mathrm{MHz}$ & $355 \mathrm{kHz}$ & $4.4 \mathrm{MHz}$ \\
\cline { 2 - 6 } & Simulation & $2.2 \mathrm{MHz}$ & $2.8 \mathrm{MHz}$ & $337 \mathrm{kHz}$ & $3.8 \mathrm{MHz}$ \\
\hline
\end{tabular}

simulation results for $V_{m e m}^{\prime}$ as a function of $V_{m e m}$ during switching up-to time $t_{\text {tran }}=100 \mu \mathrm{s}$. This is shown for two back-gate bias conditions which result in minimum and maximum spiking frequency. As seen, $V^{\prime}{ }_{m e m}$ is almost constant from point $\mathrm{A}$ to $\mathrm{B}$ and, then, changes at membrane voltage of close to $V_{d d} / 2=0.15 \mathrm{~V}$ which is taken as the spiking threshold. Table I compares the spiking frequency calculated by Eq. (5) with the simulation results for four different bias sets (cases 1-4). From this table, there is an excellent fit between the theoretical analysis and simulation. Plotted in Fig. 6 also illustrates the modulation of the spiking frequency for two back-gate biases $V_{b g 5}$ and $V_{b g 6}$ swept from $0 \mathrm{~V}$ to $1.2 \mathrm{~V}$.

\section{Monte-Carlo Analysis}

To validate the performance of the neuron in terms of mismatch, we performed the Monte-Carlo (M-C) analysis with 200 runs for the firing rate of the neuron. Figures 7(a) and (b), respectively, represent the variations of the maximum and minimum spiking-frequencies with the deviations lower than the standard deviation $3 \sigma$. This variability is because of the subthreshold operation of the transistors used in neuron circuit.

\section{Post-Layout Results and Discussion}

\section{A. Layout and the process-variation analysis}

The 22nm FDSOI process has many flavors of FETs. After performing I-V simulations of different flavors it become clear that high-threshold FETs (HVTFETs) offer the lowest leakage current as compared to other flavors of FETs in the process. HVTFET is selected to design the neuron circuit. FET 


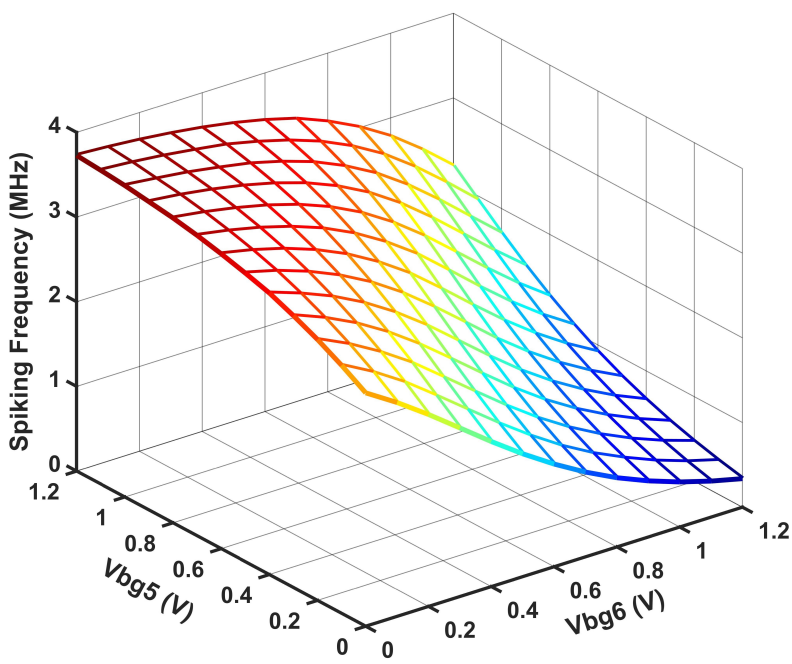

Fig. 6. Variation of the spiking frequency as a function of two back-gate biases $V_{b g 5}$ and $V_{b g 6}$.

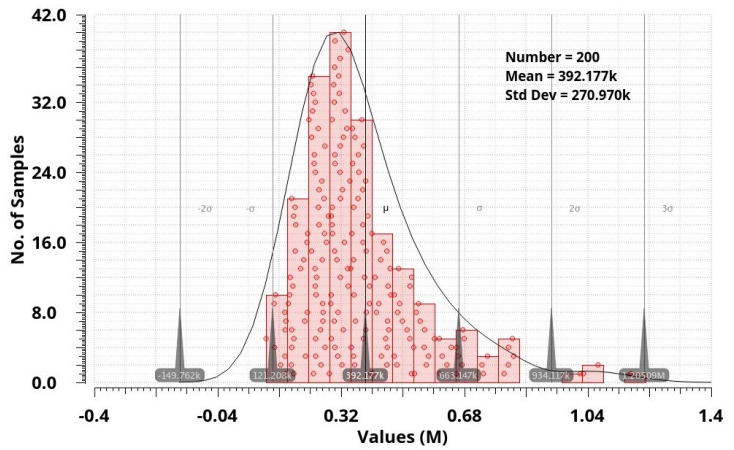

(a)

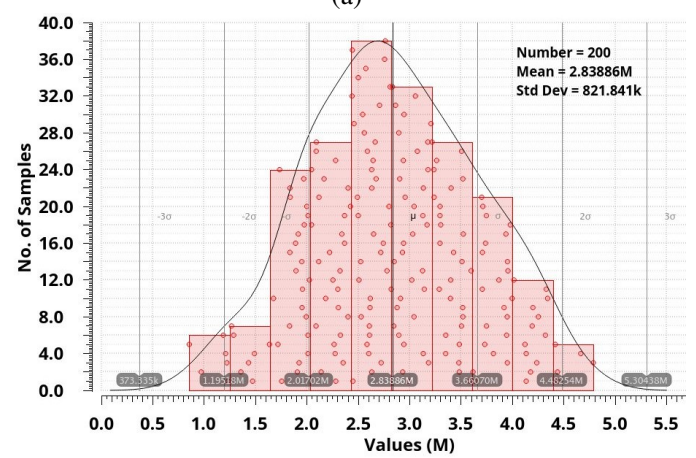

(b)

Fig. 7. The Monte-Carlo analysis for (a) the minimum and (b) maximum spiking frequencies.

widths are optimized with respect to minimize mismatch. The triple well structure, e.g. PMOS in NWELL and NMOS in PWELL, is used for all FETs. This structure allows us to isolate the NMOS device from the global substrate through an NWELL ring surrounding the device and facilitates applying an independent bias to the back-gate node [43]. An n-type current mirror with an input pulse generates the pre-synaptic current $I_{\text {syn }}$. Two 4-bit resistor-string digital-to-analog converters (DACs) are also designed to generate the two backgate biases used in the neuron circuit in Fig. 1. These DACs

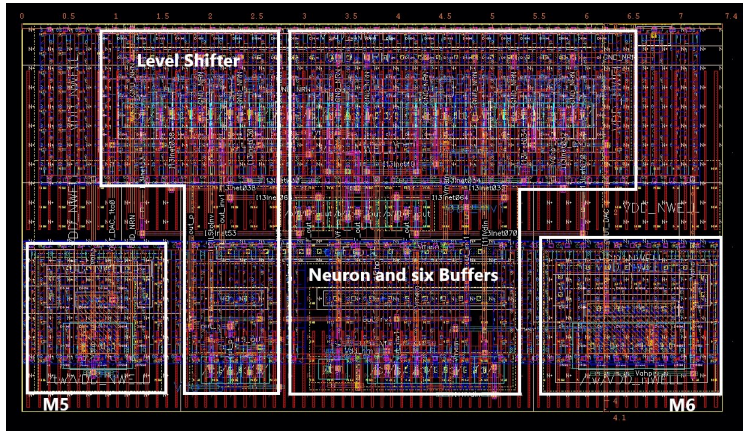

Fig. 8. The final layout including the proposed neuron in Fig. 1, the levelshifter, and all buffers needed for measurement. All capacitors are placed on top of the transistors layout, offering a more compact layout.

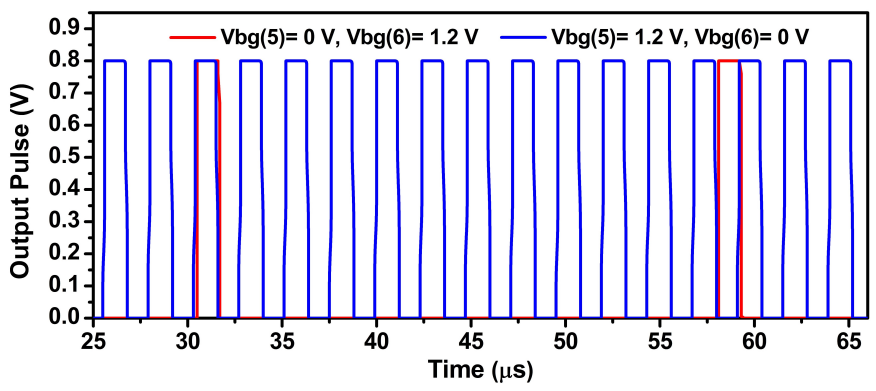

Fig. 9. The output characteristics of the level-shifter for $f_{\max }$ (blue) and $f_{\text {min }}($ red).

TABLE II

CAPACITIVE CORNER-ANALYSIS OF THE NEURON AND COMPARISON THE RESULT WITH THE NOMINAL DESIGN

\begin{tabular}{|c|c|c|c|}
\hline Corners & $f_{\min }(k \mathrm{~Hz})$ & $f_{\max }(k \mathrm{~Hz})$ & $\frac{f_{\max }}{f_{\min }}$ \\
\hline$C_{\min }$ & 38.28 & 441 & 11.52 \\
\hline Nominal & 36.8 & 416.67 & 11.32 \\
\hline$C_{\max }$ & 35.52 & 416.67 & 11.73 \\
\hline
\end{tabular}

are added to the neuron in the final layout but their areas are excluded from the neuron layout size for a fair comparison with the literature. A level-Shifter circuit [44] is inserted in the neuron layout to generate the output pulses with amplitudes of $0.8 \mathrm{~V}$ needed for the following I/O block. In addition, six output buffers are added for measurement purposes [45].

The Metal-Oxide-Metal (MOM) capacitors are chosen for the capacitors of the circuits as they offer acceptable capacitance density [43]. The final layout is presented in Fig. 8. Transistors with back-gate bias $M_{5}$ and $M_{6}$, level shifter, and other parts of neuron and buffers are shown in this figure. Total area is $30 \mu \mathrm{m}^{2}$ in size considering all blocks and MOM capacitors. Figure 9 shows the output of the level-shifter for the minimum and maximum firing rates. Although the spiking frequency is reduced by the parasitic components [39], [51], [52], the proposed neuron offers a wide frequency range from $36.8 \mathrm{kHz}$ to $416.67 \mathrm{kHz}$. The capacitors must take the values to trade-off the area and operating in the real time constants. However, small capacitors in the order of few femto-Farads are vulnerable to the parasitics. We run post-layout simulations for two extreme capacitor corners in PDK $\left(C_{\min }\right.$ and $\left.C_{\max }\right)$ and compare the ratio between $f_{\max }$ and $f_{\min }$ with the nominal 
TABLE III

COMPARISON BETWEEN THE PERFORMANCE OF THE PROPOSED NEURON WITH OTHER TOPOLOGIES AND TECHNOLOGIES REPORTED IN THE LITERATURE

\begin{tabular}{|c|c|c|c|c|c|c|c|c|c|}
\hline \multicolumn{2}{|c|}{ Reference } & Type & Technology & No. Transistors & $V_{d d}(V)$ & Power & $E_{s}$ & Frequency & Area \\
\hline \multicolumn{2}{|l|}{ [40] } & $M S$ & $0.35 \mu \mathrm{m} C M O S$ & 21 & 3.3 & - & $900 p J$ & $100 \mathrm{~Hz}$ & $<10 \mathrm{~mm}^{2} *$ \\
\hline \multicolumn{2}{|l|}{$[24]$} & $A$ & $0.35 \mu \mathrm{m} C M O S$ & 14 & 3.2 & $8-40 \mu W$ & $8.5-9 p J$ & - & $2800 \mu m^{2}$ \\
\hline \multicolumn{2}{|l|}{ [37] } & $A$ & $1.5 \mu \mathrm{m} C M O S$ & 20 & 5 & $0.3-1.5 \mu W$ & $3-15 p J$ & $100 \mathrm{~Hz}$ & - \\
\hline \multicolumn{2}{|l|}{$[1]$} & $M S$ & $28 \mathrm{~nm} \mathrm{FDSOI}$ & 21 & 1 & - & $50 p J * *$ & $30 \mathrm{~Hz}$ & $50 \mu m^{2}$ \\
\hline \multicolumn{2}{|l|}{ [5] } & $A$ & $0.18 \mu \mathrm{m} C M O S$ & 21 & 1.8 & - & $883 p J$ & $30 \mathrm{~Hz}$ & $1188 \mu m^{2}$ \\
\hline \multicolumn{2}{|l|}{ [39] } & $M S$ & $28 \mathrm{~nm} C M O S$ & 22 & $0.7-1$ & $1.9 \mathrm{~mW} \dagger$ & $2.3-30 n J$ & $1-100 \mathrm{kHz}$ & $64.6 \mu m^{2}$ \\
\hline \multicolumn{2}{|l|}{ [13] } & $A$ & $0.18 \mu m C M O S$ & 19 & 1.8 & $2-50 \mu W$ & - & - & - \\
\hline \multicolumn{2}{|l|}{ [46] } & $A$ & $C M O S$ & $27-30$ & 5 & $60 \mu W$ & - & - & - \\
\hline \multicolumn{2}{|l|}{ [47] } & $A$ & $0.35 \mu \mathrm{m} C M O S$ & 21 & 3.3 & - & $7 p J$ & - & - \\
\hline \multicolumn{2}{|l|}{ [48] } & $A$ & $0.35 \mu m T S M C$ & $>32$ & - & $3.2 n W$ & $45.7 \mathrm{pJ}$ & $70 \mathrm{~Hz}$ & $0.108 \mathrm{~mm}^{2}$ \\
\hline \multicolumn{2}{|l|}{$[22]$} & $A, D$ & $65 \mathrm{~nm} T S M C$ & $6 \dagger \dagger$ & 0.2 & $100 \mathrm{pW}$ & $4 f J$ & $25 \mathrm{kHz}$ & $35 \mu m^{2}$ \\
\hline \multicolumn{2}{|l|}{ [49] } & $A$ & $0.35 \mu \mathrm{m} \mathrm{CMOS}$ & 8 & - & $1.74 \mu W$ & $17.4 p J$ & $100 \mathrm{~Hz}$ & $1887 \mu m^{2}$ \\
\hline \multicolumn{2}{|l|}{$[12]$} & $A$ & $65 \mathrm{~nm} \mathrm{CMOS}$ & $>19$ & - & $78.16 n W$ & $41.2 \mathrm{pJ}$ & $1.9 \mathrm{MHz}$ & $538 \mu m^{2}$ \\
\hline \multicolumn{2}{|l|}{$[23]$} & $A, D$ & $90 \mathrm{~nm} C M O S$ & 14 & 0.6 & $40.2 p W$ & $0.4 p J$ & $100 \mathrm{~Hz}$ & $442 \mu m^{2}$ \\
\hline \multicolumn{2}{|l|}{$[6]$} & $M S$ & $28 \mathrm{~nm} \mathrm{FDSOI}$ & 2 & - & - & $35 f J$ & $56 \mathrm{kHz}$ & $12 \mu m^{2}$ \\
\hline \multicolumn{2}{|l|}{ [7] } & $M S$ & $28 \mathrm{~nm} T S M C$ & $5 \dagger \dagger$ & 0.2 & $30 p W$ & $2 f J$ & $15.6 \mathrm{kHz}$ & $31 \mu m^{2}$ \\
\hline \multicolumn{2}{|l|}{ [11] } & $A$ & $28 \mathrm{~nm} C M O S$ & - & 1.2 & - & $35 p J$ & $100 \mathrm{MHz}$ & $1.99 \mathrm{~mm}^{2} \ddagger$ \\
\hline \multicolumn{2}{|l|}{$[21]$} & $D$ & $0.35 \mu \mathrm{m}$ AMS CMOS & 21 & 3.3 & - & $7 p J$ & - & $913 \mu m^{2}$ \\
\hline \multicolumn{2}{|l|}{$[16]$} & $D$ & $28 \mathrm{~nm} F D S O I$ & - & $0.4-0.8$ & $12-49 n W$ & $64-280 f J$ & $16.9-524 \mathrm{MHz}$ & $574.2 \mu m^{2}$ \\
\hline \multirow{2}{*}{\multicolumn{2}{|c|}{$[50]$}} & \multirow{2}{*}{$M S$} & \multirow{2}{*}{$22 \mathrm{~nm} F D S O I$} & \multirow{2}{*}{48} & \multirow{2}{*}{0.8} & \multirow{2}{*}{-} & $14 p J$ & $30 H z$ & \multirow{2}{*}{$4800 \mu m^{2} \ddagger \ddagger$} \\
\hline & & & & & & & $850 \mathrm{fJ}$ & $1 k H z$ & \\
\hline \multirow{2}{*}{ This Work } & LF & $M S$ & $22 \mathrm{~nm} F D S O I$ & 9 & 0.3 & $1.22 n W$ & $32.36 \mathrm{fJ}$ & $36.8 \mathrm{kHz}$ & \multirow{2}{*}{$30 \mu m^{2}$} \\
\hline & $\mathrm{HF}$ & & & & & $4.7 n W$ & $11.28 f J$ & $416.67 \mathrm{kHz}$ & \\
\hline
\end{tabular}

A: Analog Implementation, D: Digital Implementation, MS: Mixed-signal Implementation, LF: Low Frequency, HF: High Frequency, $*$ Total area for 32 neurons and 8000 synapses, $* *$ Energy per spike of the whole processor, $\dagger$ Power consumption of the whole neuromorphic system, $\dagger \dagger$ These topologies lack the frequency-adaptation mechanism, $\ddagger 192$ neurons and 6144 synapses, $\ddagger \ddagger$ Tonal area for an array of four neurons and four synapses.

case. The results are summarized in Table II denoting that the frequency ratio remains roughly similar to the nominal case. This means that the capacitors are designed correctly.

\section{B. Energy Dissipation}

The energy consumed per spike is an important figure of merit (FoM) for neuron circuit. This FoM has been considered in various works to fairly evaluate the energy efficiency of the neuron circuits [6], [21], [22], [24]. Energy per spike is calculated by dividing the average power consumption $P_{\text {ave }}$ by the spiking frequency $f_{\text {spike. }}$. The averaged power is given by,

$$
P_{a v e}=\frac{1}{t_{\text {sim }}} \int_{0}^{t_{\text {sim }}} i_{d d}(t) \cdot V_{d d} d t
$$

or, equivalently,

$$
P_{\text {ave }}=\frac{f_{\text {spike }}}{N} \int_{0}^{t_{\text {sim }}} i_{d d}(t) \cdot V_{d d} d t
$$

where $t_{\text {sim }}$ is the simulation time, $i_{d d}(t)$ represents the total (static and dynamic) current flowing through the supply voltage $V_{d d}$, and $\mathrm{N}$ indicates the number of spikes in the simulation time $t_{\text {sim }}$. The energy per spike $E_{s}$ is, then, extracted from Eq. (7) and is written as

$$
E_{s}=\frac{P_{\text {ave }}}{f_{\text {spike }}}=\frac{1}{N} \int_{0}^{t_{\text {sim }}} i_{d d}(t) \cdot V_{d d} d t
$$

The averaged (static and dynamic) power consumption of the neuron at $f_{\min }=36.8 \mathrm{kHz}$ and $f_{\min }=416.67 \mathrm{kHz}$ are, respectively, $1.22 n W$ and $4.7 n W$ that correspond to $E_{s}$ of $33 \mathrm{fj} / \mathrm{s}$ and $11.28 \mathrm{fj} / \mathrm{s}$. Considering the energy per spike consumed by the level-shifter, the total energy per spike $E_{t o t}$ of the neuron and level-shifter is equal to $96.64 f j$ at $f_{\max }$ and $161 f j$ at $f_{m i n}$. Table III compares the performance of the proposed neuron with other works. Our design compares well with other silicon neurons in the literature particularly with regards to the energy consumption and the layout size. In addition, it allows frequency modulation in a broad range which is promising for larger SNN systems.

\section{CONCLUSION}

In this paper we have presented an Integrate and Fire neuron designed in $22 \mathrm{~nm}$ FDSOI technology. The proposed circuit allows spiking frequency modulation. For the first time we 
propose that the back-gate bias of the FDSOI technology can be used to create this desirable tunability. The performance of the neuron was validated through analytical models, pre, and post-layout simulations. The proposed adaptive neuron consumes an ultra-low energy with a small layout size.

\section{ACKNOWLEDGMENT}

The authors would like to thank Perceptia Device Company (https://perceptia.com/) for providing the PDK of the 22-nm FDSOI technology.

\section{REFERENCES}

[1] N. Qiao and G. Indiveri, "Scaling mixed-signal neuromorphic processors to $28 \mathrm{~nm}$ fd-soi technologies," in 2016 IEEE Biomedical Circuits and Systems Conference (BioCAS). IEEE, 2016, pp. 552-555.

[2] J. H. Wijekoon and P. Dudek, "Spiking and bursting firing patterns of a compact vlsi cortical neuron circuit," in 2007 International Joint Conference on Neural Networks. IEEE, 2007, pp. 1332-1337.

[3] J. Grollier, D. Querlioz, K. Camsari, K. Everschor-Sitte, S. Fukami, and M. D. Stiles, "Neuromorphic spintronics," Nature electronics, vol. 3, no. 7, pp. 360-370, 2020.

[4] J. H. Wijekoon and P. Dudek, "Simple analogue vlsi circuit of a cortical neuron," in 2006 13th IEEE International Conference on Electronics, Circuits and Systems. IEEE, 2006, pp. 1344-1347.

[5] K. I. Papadimitriou, S.-C. Liu, G. Indiveri, and E. M. Drakakis, "Neuromorphic log-domain silicon synapse circuits obey bernoulli dynamics: a unifying tutorial analysis," Frontiers in neuroscience, vol. 8, p. 428, 2015.

[6] T. Bédécarrats, C. Fenouillet-Béranger, S. Cristoloveanu, and P. Galy, "A bimos-based 2t1c analogue spiking neuron circuit integrated in $28 \mathrm{~nm}$ fd-soi technology for neuromorphic application," Solid-State Electronics, vol. 168, p. 107717, 2020.

[7] F. Danneville, C. Loyez, K. Carpentier, I. Sourikopoulos, E. Mercier, and A. Cappy, "A sub-35 pw axon-hillock artificial neuron circuit," SolidState Electronics, vol. 153, pp. 88-92, 2019.

[8] N. K. Upadhyay, H. Jiang, Z. Wang, S. Asapu, Q. Xia, and J. Joshua Yang, "Emerging memory devices for neuromorphic computing," Advanced Materials Technologies, vol. 4, no. 4, p. 1800589, 2019.

[9] P. U. Diehl and M. Cook, "Unsupervised learning of digit recognition using spike-timing-dependent plasticity," Frontiers in computational neuroscience, vol. 9, p. 99, 2015.

[10] T. J. Hamilton, S. Afshar, A. van Schaik, and J. Tapson, "Stochastic electronics: A neuro-inspired design paradigm for integrated circuits," Proceedings of the IEEE, vol. 102, no. 5, pp. 843-859, 2014.

[11] Y. Liu, K. Qian, S. Hu, K. An, S. Xu, X. Zhan, J. Wang, R. Guo, Y. Wu, T.-P. Chen et al., "Application of deep compression technique in spiking neural network chip," IEEE transactions on biomedical circuits and systems, vol. 14, no. 2, pp. 274-282, 2019.

[12] A. Joubert, B. Belhadj, O. Temam, and R. Héliot, "Hardware spiking neurons design: Analog or digital?" in The 2012 International Joint Conference on Neural Networks (IJCNN). IEEE, 2012, pp. 1-5.

[13] E. Radhika, S. Kumar, and A. Kumari, "Low power analog vlsi implementation of cortical neuron with threshold modulation," in 2015 International Conference on Advances in Computing, Communications and Informatics (ICACCI). IEEE, 2015, pp. 561-566.

[14] C. Frenkel, M. Lefebvre, J.-D. Legat, and D. Bol, "A 0.086-mm 212.7 pj/sop 64k-synapse 256-neuron online-learning digital spiking neuromorphic processor in 28-nm cmos," IEEE transactions on biomedical circuits and systems, vol. 13, no. 1, pp. 145-158, 2018.

[15] G. Indiveri, F. Stefanini, and E. Chicca, "Spike-based learning with a generalized integrate and fire silicon neuron," in Proceedings of 2010 IEEE International Symposium on Circuits and Systems. IEEE, 2010, pp. 1951-1954.

[16] C. Frenkel, J.-D. Legat, and D. Bol, "A compact phenomenological digital neuron implementing the 20 izhikevich behaviors," in 2017 IEEE Biomedical Circuits and Systems Conference (BioCAS). IEEE, 2017, pp. 1-4.

[17] G. Indiveri, B. Linares-Barranco, T. J. Hamilton, A. Van Schaik, R. Etienne-Cummings, T. Delbruck, S.-C. Liu, P. Dudek, P. Häfliger, S. Renaud et al., "Neuromorphic silicon neuron circuits," Frontiers in neuroscience, vol. 5, p. 73, 2011.
[18] N. Qiao and G. Indiveri, "Analog circuits for mixed-signal neuromorphic computing architectures in $28 \mathrm{~nm}$ fd-soi technology," in 2017 IEEE SOI3D-Subthreshold Microelectronics Technology Unified Conference (S3S). IEEE, 2017, pp. 1-4.

[19] N. Qiao, H. Mostafa, F. Corradi, M. Osswald, F. Stefanini, D. Sumislawska, and G. Indiveri, "A reconfigurable on-line learning spiking neuromorphic processor comprising 256 neurons and 128k synapses," Frontiers in neuroscience, vol. 9, p. 141, 2015.

[20] C. Lee, G. Srinivasan, P. Panda, and K. Roy, "Deep spiking convolutional neural network trained with unsupervised spike-timing-dependent plasticity," IEEE Transactions on Cognitive and Developmental Systems, vol. 11 , no. 3, pp. 384-394, 2018.

[21] P. Livi and G. Indiveri, "A current-mode conductance-based silicon neuron for address-event neuromorphic systems," in 2009 IEEE international symposium on circuits and systems. IEEE, 2009, pp. 2898-2901.

[22] I. Sourikopoulos, S. Hedayat, C. Loyez, F. Danneville, V. Hoel, E. Mercier, and A. Cappy, "A 4-fj/spike artificial neuron in $65 \mathrm{~nm}$ cmos technology," Frontiers in neuroscience, vol. 11, p. 123, 2017.

[23] J. M. Cruz-Albrecht, M. W. Yung, and N. Srinivasa, "Energy-efficient neuron, synapse and stdp integrated circuits," IEEE transactions on biomedical circuits and systems, vol. 6, no. 3, pp. 246-256, 2012.

[24] J. H. Wijekoon and P. Dudek, "Compact silicon neuron circuit with spiking and bursting behaviour," Neural Networks, vol. 21, no. 2-3, pp. 524-534, 2008.

[25] C. Mead, Analog VLSI and neural systems. Addison-Wesley Longman Publishing Co., Inc., 1989.

[26] A. L. Hodgkin and A. F. Huxley, "A quantitative description of membrane current and its application to conduction and excitation in nerve," The Journal of physiology, vol. 117, no. 4, pp. 500-544, 1952.

[27] C. Morris and H. Lecar, "Voltage oscillations in the barnacle giant muscle fiber," Biophysical journal, vol. 35, no. 1, pp. 193-213, 1981.

[28] R. FitzHugh, "Impulses and physiological states in theoretical models of nerve membrane," Biophysical journal, vol. 1, no. 6, pp. 445-466, 1961.

[29] K. Nakada, T. Asai, and Y. Amemiya, "Analog cmos implementation of a bursting oscillator with depressing synapse," in Proceedings of the 2004 Intelligent Sensors, Sensor Networks and Information Processing Conference, 2004. IEEE, 2004, pp. 503-506.

[30] K. Nakada, T. Asai, and H. Hayashi, "A silicon resonate-and-fire neuron based on the volterra system," in Int. symp. on nonlinear theory and its applications, 2005, pp. 82-85.

[31] Y. J. Lee, J. Lee, Y.-B. Kim, J. Ayers, A. Volkovskii, A. Selverston, H. Abarbanel, and M. Rabinovich, "Low power real time electronic neuron vlsi design using subthreshold technique," in 2004 IEEE International Symposium on Circuits and Systems (IEEE Cat. No. 04CH37512), vol. 4. IEEE, 2004, pp. IV-744.

[32] E. M. Izhikevich, "Simple model of spiking neurons," IEEE Transactions on neural networks, vol. 14, no. 6, pp. 1569-1572, 2003.

[33] F. Folowosele, A. Harrison, A. Cassidy, A. G. Andreou, R. EtienneCummings, S. Mihalas, E. Niebur, and T. J. Hamilton, "A switched capacitor implementation of the generalized linear integrate-and-fire neuron," in 2009 IEEE International Symposium on Circuits and Systems (ISCAS). IEEE, 2009, pp. 2149-2152.

[34] F. Folowosele, R. Etienne-Cummings, and T. J. Hamilton, "A cmos switched capacitor implementation of the mihalas-niebur neuron," in 2009 IEEE Biomedical Circuits and Systems Conference. IEEE, 2009, pp. $105-108$.

[35] A. Van Schaik, C. Jin, A. McEwan, T. J. Hamilton, S. Mihalas, and E. Niebur, "A log-domain implementation of the mihalas-niebur neuron model," in Proceedings of 2010 IEEE International Symposium on Circuits and Systems. IEEE, 2010, pp. 4249-4252.

[36] F. Folowosele, T. J. Hamilton, and R. Etienne-Cummings, "Silicon modeling of the mihalaş-niebur neuron," IEEE transactions on neural networks, vol. 22, no. 12, pp. 1915-1927, 2011.

[37] G. Indiveri, "A low-power adaptive integrate-and-fire neuron circuit," in Proceedings of the 2003 International Symposium on Circuits and Systems, 2003. ISCAS'03., vol. 4. IEEE, 2003, pp. IV-IV.

[38] R. M. Wang, T. J. Hamilton, J. C. Tapson, and A. van Schaik, "A neuromorphic implementation of multiple spike-timing synaptic plasticity rules for large-scale neural networks," Frontiers in neuroscience, vol. 9, p. $180,2015$.

[39] C. Mayr, J. Partzsch, M. Noack, S. Hänzsche, S. Scholze, S. Höppner, G. Ellguth, and R. Schüffny, "A biological-realtime neuromorphic system in $28 \mathrm{~nm}$ cmos using low-leakage switched capacitor circuits," IEEE transactions on biomedical circuits and systems, vol. 10, no. 1, pp. 243254, 2015. 
[40] G. Indiveri, E. Chicca, and R. Douglas, "A vlsi array of low-power spiking neurons and bistable synapses with spike-timing dependent plasticity," IEEE transactions on neural networks, vol. 17, no. 1, pp. 211-221, 2006.

[41] D. Rajasekharan, P. Kushwaha, S. S. Chauhan, and Y. S. Chauhan, "Nonboolean associative processing using fdsoi mosfet-based inverter," IEEE Transactions on Nanotechnology, vol. 17, no. 6, pp. 1235-1243, 2018.

[42] A. Carbon, J.-M. Philippe, O. Bichler, R. Schmit, B. Tain, D. Briand, N. Ventroux, M. Paindavoine, and O. Brousse, "Pneuro: A scalable energy-efficient programmable hardware accelerator for neural networks," in 2018 Design, Automation \& Test in Europe Conference \& Exhibition (DATE). IEEE, 2018, pp. 1039-1044.

[43] L. Le Guevel, G. Billiot, B. Cardoso Paz, M. Tagliaferri, S. De Franceschi, R. Maurand, M. Cassé, M. Zurita, M. Sanquer, M. Vinet et al., "Low-power transimpedance amplifier for cryogenic integration with quantum devices," Applied Physics Reviews, vol. 7 , no. 4, p. 041407, 2020.

[44] S. Lütkemeier and U. Rückert, "A subthreshold to above-threshold level shifter comprising a wilson current mirror,' IEEE Transactions on Circuits and Systems II: Express Briefs, vol. 57, no. 9, pp. 721-724, 2010.

[45] M. Franco, J. Güiza, E. Chiappetta, S. Rueda, H. Luis, J. Bertuzzo, J. Koeppe, T. Robins, J. Jenkins, and T. Hamilton, "Electronically programmable test points for on-chip analog/digital measurements," in 2013 IEEE International Symposium on Circuits and Systems (ISCAS). IEEE, 2013, pp. 2670-2673.

[46] M. Mahowald and R. Douglas, "A silicon neuron," Nature, vol. 354, no. 6354, pp. 515-518, 1991.

[47] M. Ranjbar and M. Amiri, "Analog implementation of neuron-astrocyte interaction in tripartite synapse," Journal of Computational Electronics, vol. 15, no. 1, pp. 311-323, 2016.

[48] T. Kohno, M. Sekikawa, J. Li, T. Nanami, and K. Aihara, "Qualitativemodeling-based silicon neurons and their networks," Frontiers in neuroscience, vol. 10, p. 273, 2016.

[49] A. Basu and P. E. Hasler, "Nullcline-based design of a silicon neuron," IEEE Transactions on Circuits and Systems I: Regular Papers, vol. 57, no. 11 , pp. 2938-2947, 2010.

[50] A. Rubino, C. Livanelioglu, N. Qiao, M. Payvand, and G. Indiveri, "Ultra-low-power fdsoi neural circuits for extreme-edge neuromorphic intelligence," IEEE Transactions on Circuits and Systems I: Regular Papers, vol. 68, no. 1, pp. 45-56, 2020.

[51] K. I. Papadimitriou and E. M. Drakakis, "Cmos weak-inversion logdomain glycolytic oscillator: a cytomimetic circuit example," International Journal of Circuit Theory and Applications, vol. 42, no. 2, pp. 173-194, 2014.

[52] B. Razavi, Design of analog CMOS integrated circuits. Tata McGrawHill Education, 2002. 